\title{
PROJEÇÕES CLIMÁTICAS REGIONALIZADAS PARA O ESTADO DO TOCANTINS, BRASIL, NOS CENÁRIOS RCP 4.5 E RCP 8.5
}

\author{
SOUSA, Rhonan Martins de - rohsousa@hotmail.com \\ Universidade Federal do Tocantins / UFT
}

VIOLA, Marcelo Ribeiro - marcelo.viola@deg.ufla.br Universidade Federal de Lavras / UFLA

CHOU, Sin Chan - chou.sinchan@gmail.com

Instituto Nacional de Pesquisas Espaciais / INPE

ALVES, Marcos Vinicius Giongo - giongo@uft.edu.br Universidade Federal do Tocantins / UFT

AVANZI ,Junior Cesar - junior.avanzi@ufla.br Universidade Federal de Lavras / UFLA

RESUMO: Os possíveis impactos sobre os recursos hídricos e a biodiversidade do Cerrado decorrentes da alteração de origem antrópica da concentração de Gases de Efeito Estufa (GEEs) é tida como uma das principais problemáticas ambientais do século XXI. O avanço de pesquisas sobre essa temática é realizado a partir da aplicação de modelos climáticos, que possibilitam a avaliação de cenários exploratórios futuros. Nesse contexto, objetivou-se avaliar as mudanças climáticas projetadas pelo modelo climático regional Eta dirigido pelos modelos globais HadGEM2-ES e MIROC5 para o início (2011-2040) e metade (2041-2070) do século XXI no Estado do Tocantins, para as trajetórias representativas de concentração, RCP 4.5 e RCP 8.5. Levando-se em consideração o regime pluvial inerente ao Tocantins, os resultados foram analisados para as estações chuvosa (de outubro a março) e estiagem (de abril a setembro), tendo sido abordadas as seguintes variáveis: precipitação, evapotranspiração real, temperaturas (média, máxima e mínima) e umidade relativa. A análise das projeções climáticas futuras mostrou possibilidade de severas alterações nos regimes hídrico e térmico no estado do Tocantins nos RCPs 4.5 e 8.5 no século XXI. As projeções de mudanças mais severas de precipitação e temperatura foram pelo Eta-MIROC5 e Eta-HadGEM2-ES, respectivamente, ambas no cenário RCP 8.5. As projeções mostram possibilidade de redução da precipitação de até $524,8 \mathrm{~mm}^{-6 \mathrm{meses}^{-1}}$ (out-mar) para o início do século XXI, sobretudo para a região Sul. Para as temperaturas (média, mínima e máxima) foram projetadas mudanças positivas em todas as análises. As mudanças mais severas foram para a metade do século XXI, de até $5,1^{\circ} \mathrm{C}$ para as temperaturas médias, $5,9^{\circ} \mathrm{C}$ para as temperaturas máximas e $4,8^{\circ} \mathrm{C}$ para as temperaturas mínimas, sobretudo para a região Sudoeste nas proximidades da Ilha do Bananal.

Palavras-chave: Modelo climático regional, Cerrado brasileiro, cenário de mudanças climáticas

DOWNSCALED CLIMATE PROJECTIONS OVER TOCANTINS STATE, BRAZIL, UNDER RCP 4.5 AND RCP 8.5 SCENARIOS.

ABSTRACT: The possible impacts on the water resources and the biodiversity of the Cerrado due to the anthropogenic concentration of Greenhouse Gases (GHGs) is 
considered as one of the main environmental problems of the $21_{\text {st }}$ century. The advance of research on this theme is carried out through the application of climate models, which allow the evaluation of future climate scenarios. In this context, the objective was to asses the climatic changes projected by the regional climatic model Eta, driven by the global models HadGEM2-ES and MIROC5 for the early (2011-2040) and mid (2041-2070) of the $21_{\text {st }}$ century, in the State of Tocantins, for the Representative Concentration Pathways (RCPs) 4.5 and 8.5. The results were analyzed for the rainy season (October to March) and dry season (April to September), with the following variables being considered: rainfall, evapotranspiration, temperature (mean, maximum and minimum) and relative humidity. The analysis of the future projections exposed the change possibility in the water and thermal regimes in the State of Tocantins in RCPs 4.5 and 8.5 for the $21_{\text {st }}$ century. The most severe projections for rainfall and temperature were provided by Eta-MIROC5 and Eta-HadGEM2-ES, respectively, both for the RCP 8.5 scenary The projections show possibility of reduction of rainfall of up to 524,8 mm.6months ${ }^{-1}$ (Oct-Mar) for the begining of the 21 st century, mostly for the South region of the State. For the temperatures (mean, maximum and minimum), positive changes were projected for all analyzes. The most severe changes were projected for the mid of the 21 st century, of up to $5.1^{\circ} \mathrm{C}$ (mean temp.), $5.9^{\circ} \mathrm{C}$ (maximum temp.) and 4.8 ${ }^{\circ} \mathrm{C}$ (minimum temp.), especially for the Southwest region, near the Bananal Island.

Keywords: Regional climate model, Brazilian Cerrado, climate change scenarios

\section{INTRODUÇÃO}

O Cerrado é o maior bioma totalmente inserido em território brasileiro, ocupando cerca de 204,7 milhões de hectares, o que corresponde a $24 \%$ da área nacional (IBGE, 2004). Levando em consideração a diversidade biológica, 0 Cerrado brasileiro varia bastante em sua fisionomia, apresentando nos diversos ecossistemas, padrões heterogêneos de composição florística, além de permitir a ocorrência de alta riqueza de espécies (FELFILI et al., 2004; FRANÇOSO et al., 2016), com uma flora superior a 12.000 espécies de plantas nativas (MENDONÇA et al., 2008). O bioma Cerrado é caracterizado por apresentar uma vegetação influenciada pela sazonalidade e concentração de biomassa, sendo formado por exemplares arbóreos, herbáceos e arbustivos, se expressando em diversas fitofisionomias (EVANGELISTA, 2016).

O bioma Cerrado exerce um importante papel na produção e distribuição de recursos hídricos para oito das doze regiões hidrográficas brasileiras (LIMA e SILVA, 2007). Nesse contexto, o Cerrado é considerado o "berço das águas do Brasil", "pai das águas do Brasil" ou a "caixa d'água do Brasil", pois além de abrigar uma parte do aquífero Guarani, nele surgem importantes afluentes dos rios Paraná, Tocantins, São Francisco e Amazonas (LIMA, 2011). Estudos apontam que $60 \%$ da produção hídrica superficial da bacia hidrográfica do Tocantins-Araguaia tem origem na área de ocorrência do Cerrado (LIMA e SILVA, 2007).

O IPCC (Intergovernmental Panel on Climate Change) foi consolidado em 1988 pelo United Nations Environment Programme (UNEP) e pela World Meteorological Organization (WMO), sendo considerado o principal órgão científico responsável pelas avaliações das mudanças climáticas e seus impactos ambientais e socioeconômicos (IPCC, 2007). De acordo com o IPCC mudanças climáticas consistem em variações estatisticamente significativas de variáveis representativas do clima tais como precipitação, temperatura, entre outras. Estas mudanças podem ser ocasionadas por processos naturais, forçamentos externos, e até mesmo por ações antrópicas na composição do uso do solo e da atmosfera (IPCC, 2013). Ressalta-se que, o acúmulo de Gases de Efeito Estufa 
(GEEs) ao longo das últimas décadas tem sido intensificado por atividades humanas, refletindo em mudanças climáticas de origem antrópica (IPCC, 2013; DELAZERI, 2015).

Cubash et al. (2013) destacam que as informações mais recentes sobre as mudanças climáticas globais são encontradas no Quinto Relatório de Avaliação (AR5) do IPCC. O AR5 se baseia nos Representative Concentration Pathways (RCPs), que consistem em projeções das concentrações de Gases de Efeito Estufa (GEEs), gases quimicamente ativos, aerossóis e uso e cobertura do solo para o século XXI (VAN VUUREN et al., 2011) e seu consequente aumento da retenção de energia no planeta, denominado de forçante radiativa e mensurado em $\mathrm{Wm}^{-2}$. Os RCPs foram criados com o intuito de servir como entrada para a modelagem química atmosférica e climática em experimentos numéricos do Coupled Model Intercomparison Project Phase 5 (CMIP5) (SILVEIRA et al., 2016). No AR5 são tratados diferentes RCPs, sendo estes nomeados em função da forçante radiativa atingida ao final do século XXI em RCP: 2.6, 4.5, 6.0 e 8.5 (MOSS et al., 2010). Desta maneira, o RCP mais severo projeta um aumento da forçante radiativa de $8,5 \mathrm{Wm}$ até o final do século XXI (RCP 8.5); enquanto que o RCP 4.5 é tido como uma projeção intermediária entre o 2.6 e o 8.5 ( $\mathrm{CHOU}$ et al. 2014a).

Estudos recentes mostram que mudanças climáticas podem afetar os processos hidrológicos em bacias hidrográficas (TAN et al., 2017), ou mesmo a distribuição de espécies e a biodiversidade (COX et al., 2004; GOLDING e BETTS, 2008; MALHI et al., 2009; WANG et al., 2012). Com o objetivo de modelar o clima global, são estruturados modelos numéricos de macroescala denominados Modelos Climáticos Globais (MCGs). Os MCGs são capazes de descrever as interações entre atmosfera, solos, vegetação e oceanos, sendo considerados importantes ferramentas para estudos sobre o clima futuro (MARENGO et al., 2012; ADAM e COLLISCHONN, 2013). Para o desenvolvimento de estudos na escala regional são utilizados Modelos Climáticos Regionais (MCRs) visando a representação dos processos físicos em menor escala, consequentemente com resultados mais detalhados (FESER et al., 2011).

O Estado do Tocantins tem área de $277.621 \mathrm{~km}^{2}(3,26 \%$ do território nacional e $7,17 \%$ da região Norte). O bioma Cerrado ocupa cerca de $91 \%$ da área do Estado, sendo os demais $9 \%$ ocupados pelo bioma Amazônia (IBGE, 2016). Resultados de pesquisas recentes desenvolvidos para toda a América do Sul (Chou et al., 2014b) e também para a escala de bacia hidrográfica (RODRIGUES, 2017) mostram projeções de mudanças climáticas importantes para a região central do Brasil, denotando a necessidade de desenvolvimento de estudos específicos para o estado do Tocantins. Tais estudos visam sobretudo subsidiar a formulação de políticas públicas para tratar sobre a prevenção e mitigação de possíveis impactos de mudanças climáticas futuras.

Neste contexto, objetivou-se avaliar as mudanças climáticas projetadas para o Estado do Tocantins pelo MCR Eta dirigido pelos MCGs HadGEM2-ES e MIROC5 nos RCPs 4.5 e 8.5, sendo denominados no presente estudo de EtaHadGEM2-ES e Eta-MIROC5, respectivamente. Especificamente objetivou-se mapear as mudanças climáticas projetadas para a precipitação, evapotranspiração real, temperatura (máxima, média e mínima) e umidade relativa, em dois períodos futuros ao longo do século XXI. 


\section{MATERIAL E MÉTODOS}

O Estado do Tocantins está localizado na região Norte do Brasil, entre os paralelos $5^{0} 10^{\prime} \mathrm{S}$ e $13^{\circ} 28^{\prime} \mathrm{S}$ e entre os meridianos $45^{\circ} 45^{\prime} \mathrm{W}$ e $50^{\circ} 44^{\prime} \mathrm{W}$. O Tocantins faz divisa com os Estados da Bahia, Goiás, Maranhão, Mato Grosso, Pará e Piauí (IBGE, 2004). A Figura 1 mostra a localização do Estado do Tocantins e os biomas. De acordo com Souza et al. (2019) ocorrem os tipos climáticos B1wA'a' (clima úmido com moderada deficiência de água no inverno) no oeste do estado, C2WA'a' (clima subúmido com moderada deficiência de água no inverno) na região central e $C 1 w 2 A^{\prime} a^{\prime}$ (clima subúmido seco, com grande excesso de água no verão) no leste e nordeste

A.

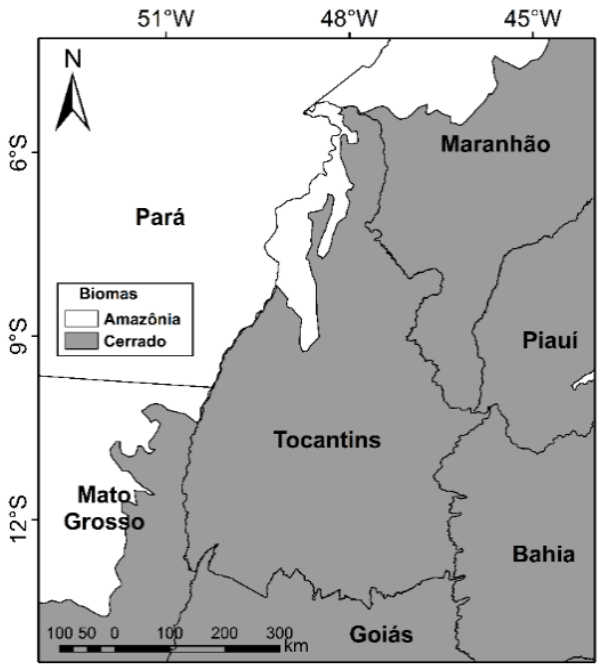

B.

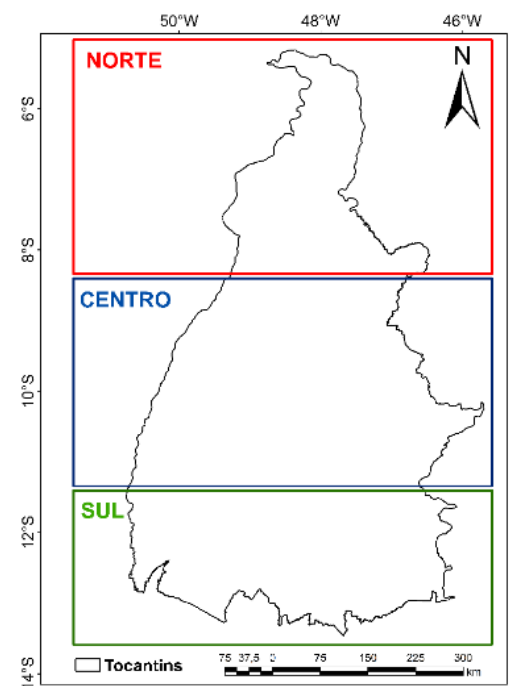

Figura 1 - Biomas e divisas estaduais (A) e regiões definidas para análise dos resultados (B).

O MCG HadGEM2-ES (Hadley Centre Global Environmental Model version 2 Earth System) (COLLINS et al., 2011; MARTIN et al., 2011) é um modelo em ponto de grade com resolução N96, equivalente a 1,875 e 1,275 graus de longitude e latitude, respectivamente, e 38 níveis verticais na atmosfera, chegando a cerca de $40 \mathrm{~km}$ de altitude. O HadGEM2-ES foi usado pelo Met Office Hadley Centre para as simulações do Coupled Model Intercomparison Project Phase 5 (CMIP5) (IPCC, 2013), que utiliza dados históricos de 1960 a 2005 e projeções através dos RCPs do AR5 (MOSS et al., 2010).

O Model for Interdisciplinary Research on Climate (MIROC5) (WATANABE et al., 2010), foi desenvolvido em conjunto pelo National Institute of Environmental Studies (NIES), Center for Climate System Research (CCSR) e Japan Agency for Marine-Earth Science and Technology (JAMSTEC), sendo essa nova versão utilizada no IPCC, no AR5 (IPCC, 2013). Segundo Watanabe et al. (2010), o componente atmosférico do MIROC5 apresenta resolução vertical de 40 níveis, com resolução T85, correspondendo a aproximadamente $150 \mathrm{~km}$ na horizontal. O componente oceânico é o Center for Climate System Research Ocean Component Model (COCO 4.5) (HASUMI, 2006), apresentando $1^{0}$ de resolução horizontal e 40 níveis atmosféricos.

O MCR utilizado neste trabalho foi o Eta, sendo desenvolvido na Universidade de Belgrado, empregado operacionalmente pelo National Centers 
for Environmental Prediction (NCEP) (BLACK, 1994); e recebendo atualizações (MESINGER et al., 2012) no Centro de Previsão de Tempo e Estudos Climáticos do Instituto Nacional de Pesquisas Espaciais (CPTEC/INPE). Atualmente é utilizado na previsão do tempo no CPTEC/INPE, e amplamente utilizado na América do Sul em estudos de previsão, em diferentes escalas de tempo e com resolução espacial de até $5 \mathrm{~km}$ na horizontal (CHOU et al., 2014a). A versão do modelo para estudos de mudanças climáticas foi desenvolvida inicialmente na resolução horizontal de 40 km (PESQUERO et al. 2010; CHOU et al. 2012; MARENGO et al. 2012) para gerar projeções no cenário A1B.

No presente estudo foram utilizadas projeções climáticas disponibilizadas por Chou et al. (2014a; 2014b) em que o modelo Eta foi dirigido pelas saídas dos modelos globais HadGEM2-ES e MIROC5, sendo denominados no presente estudo de Eta-HadGEM2-ES e Eta-MIROC5, respectivamente. Esta versão do modelo foi estruturada na resolução de $20 \mathrm{~km}, 38$ camadas na vertical e $25 \mathrm{hPa}$ na parte superior do modelo. Especificamente foram analisados os cenários de concentração dos GEE RCP 4.5 e RCP 8.5, nos períodos de 2011 a 2040 e de 2041 a 2070. O RCP 4.5 pressupõe que a forçante radiativa se estabiliza em 4,5 W. $\mathrm{m}^{-2}$ em 2100, atingindo cerca de $650 \mathrm{ppm}$ de $\mathrm{CO}_{2}$ ao fim do século XXI (THOMSON et al., 2011). Segundo Riahi et al. (2011), o RCP 8.5 estabelece as maiores emissões de gases de efeito estufa, sendo considerado uma projeção pessimista, atingindo 8,5 W. $\mathrm{m}^{-2}$ e 936 ppm de $\mathrm{CO}_{2}$ em 2100.

Para o cálculo das mudanças climáticas considerou-se como referência o clima presente simulado pelos modelos entre 1961 e 1990. Para a quantificação das mudanças climáticas futuras projetadas, consideraram-se dois períodos, 0 primeiro visando avaliar o início do século XXI (de 2011 a 2040) e o segundo para a metade do século XXI (de 2041 a 2070). Para cada período futuro, separaram-se os resultados em período chuvoso (de outubro a março) e seco (de abril a setembro). O cálculo das mudanças climáticas foi obtido pela diferença entre a média da projeção para o período futuro e a média para o clima presente. Valores positivos representam projeção de aumento da variável no período futuro, enquanto que, valores negativos representam projeção de redução. As mudanças climáticas também foram caracterizadas por região, sendo Norte, Centro e Sul (Figura 1B). Foram analisadas as seguintes variáveis climatológicas: precipitação acumulada, evapotranspiração real acumulada, temperaturas (mínima, média e máxima) e umidade relativa.

\section{RESULTADOS E DISCUSSÃO}

A Tabela 1 traz o resumo geral das mudanças climáticas agrupadas para as regiões Norte, Centro e Sul. Assim, torna-se possível de variação contemplando os dois modelos (Eta-MIROC5 e Eta-HadGEM2-ES) e os dois RCPs (4.5 e 8.5) para os períodos futuros. As Figuras 2 e 3 mostram os mapas com as projeções de mudanças climáticas para precipitação do período chuvoso e estiagem, respectivamente. 
Tabela 1 - Faixa de variação para as mudanças climáticas obtidas para as regiões Norte, Centro e Sul para os dois períodos futuros estudados, abrangendo os resultados das duas projeções (Eta-MIROC5 e Eta-HadGEM2-ES) e RCP 4.5 e RCP 8.5.

\begin{tabular}{|c|c|c|c|}
\hline \multirow{2}{*}{ Região } & \multirow{2}{*}{ Variável } & \multicolumn{2}{|c|}{ Período climático futuro } \\
\hline & & 2011-2040 & $2041-2070$ \\
\hline \multirow{6}{*}{ Norte } & Precipitação (mm.6meses $\left.{ }^{-1}\right)$ & {$[-500 ; 0]$} & {$[-429 ; 19,3]$} \\
\hline & Umidade relativa (\%) & {$[-10 ;-1,2]$} & {$[-10 ;-1,1]$} \\
\hline & Temperatura média $\left({ }^{\circ} \mathrm{C}\right)$ & {$[0,6 ; 2,8]$} & {$[1,7 ; 4,8]$} \\
\hline & Temperatura máxima $\left({ }^{\circ} \mathrm{C}\right)$ & {$[1 ; 3,6]$} & {$[1,8 ; 5,9]$} \\
\hline & Temperatura mínima ( $\left.{ }^{\circ} \mathrm{C}\right)$ & {$[0,6 ; 2,8]$} & {$[1,5 ; 4,4]$} \\
\hline & Evapotranspiração real (mm.6meses $\left.{ }^{-1}\right)$ & {$[-180 ;-30]$} & {$[-189,7 ;-4,4]$} \\
\hline \multirow{6}{*}{ Centro } & Precipitação $\left(\mathrm{mm} .6 \mathrm{meses}^{-1}\right)$ & {$[-500 ; 24,6]$} & {$[-497,7 ; 19,3]$} \\
\hline & Umidade relativa (\%) & {$[-12 ;-2]$} & {$[-13,7 ;-2]$} \\
\hline & Temperatura média $\left({ }^{\circ} \mathrm{C}\right)$ & {$[0,6 ; 3,2]$} & {$[1,7 ; 5,1]$} \\
\hline & Temperatura máxima ( $\left.{ }^{\circ} \mathrm{C}\right)$ & {$[1 ; 4,0]$} & {$[1,8 ; 5,6]$} \\
\hline & Temperatura mínima ( $\left.{ }^{\circ} \mathrm{C}\right)$ & {$[0,6 ; 2,9]$} & {$[1,6 ; 4,8]$} \\
\hline & Evapotranspiração real (mm.6meses $\left.{ }^{-1}\right)$ & {$[-206,7 ;-28,1]$} & {$[-180 ;-4,4]$} \\
\hline \multirow{6}{*}{ Sul } & Precipitação $\left(\mathrm{mm} .6 \mathrm{meses}^{-1}\right)$ & {$[-524,8 ; 24,6]$} & {$[-497,7 ; 19,3]$} \\
\hline & Umidade relativa (\%) & {$[-13,2 ;-2]$} & {$[-13,7 ;-2]$} \\
\hline & Temperatura média $\left({ }^{\circ} \mathrm{C}\right)$ & {$[0,8 ; 3,2]$} & {$[1,7 ; 5,1]$} \\
\hline & Temperatura máxima ( $\left.{ }^{\circ} \mathrm{C}\right)$ & {$[1 ; 4,1]$} & {$[1,8 ; 5,9]$} \\
\hline & Temperatura mínima ( $\left.{ }^{\circ} \mathrm{C}\right)$ & {$[0,6 ; 2,9]$} & {$[1,6 ; 4,8]$} \\
\hline & Evapotranspiração real (mm.6meses $\left.{ }^{-1}\right)$ & {$[-206,7 ;-30]$} & {$[-181,3 ;-4,4]$} \\
\hline
\end{tabular}

A Figura 2 mostra que as projeções para o total precipitado no período chuvoso tem sinal negativo para todo o Estado do Tocantins em ambos RCPs (4.5 e 8.5), ou seja, em comparação com o clima presente foi projetada redução da precipitação acumulada. Nota-se que as mudanças climáticas de precipitação geradas pelo Eta-MIROC5 e pelo RCP 8.5 foram mais severas que as do EtaHadGEM2-ES e pelo RCP 4.5, respectivamente. A maior redução foi projetada para a região Sul, de até $-524,8 \mathrm{~mm} .6$ meses $^{-1}$ (Tabela 1 ) pelo Eta-MIROC5 para o início do século XXI (2011-2040) no RCP 8.5.

Para o período de estiagem (abril-setembro) as projeções futuras também apresentaram sinal negativo (Figura 3), com exceção do Eta-HadGEM2ES no RCP 8.5 para ambos os períodos futuros, situação na qual se encontraram mudanças positivas de até $24,6 \mathrm{~mm} 6 \mathrm{meses}^{-1}$ para alguns locais, indicando maior incerteza nas projeções desse período. Rodrigues (2017) avaliou os possíveis impactos hidrológicos associados aos cenários RCP 4.5 e RCP 8.5 para três bacias hidrográficas localizadas no sudeste do Tocantins. Os resultados das mudanças climáticas futuras projetadas para as três bacias mostraram possibilidade de redução da precipitação de até 31,9\% e 29,5\% para o início (2011-2040) e meados (2041-2070) do século XXI, respectivamente, corroborando com o sinal das mudanças identificado no presente estudo.

As projeções de mudanças futuras nos cenários RCP 4.5 e RCP 8.5 para a umidade relativa do ar (Figuras 4 e 5), de forma geral, acompanham o padrão de distribuição espacial obtido para a precipitação. As faixas de variação das projeções foram de $[-13,2 \% ;-1,2 \%]$ e $[-13,7 \% ;-1,1 \%]$ para o início e meados do século XXI, respectivamente (Tabela 1 ). As projeções de mudanças mais severas foram pelo Eta-HadGEM2-ES no RCP 8.5 para meados do século XXI, 
para o sul e sudoeste do Tocantins, com gradiente decrescente em direção ao nordeste. Klink e Moreira (2002) destacaram que a redução da umidade em período de seca pode corroborar para a ocorrência de queimadas, além de degradar a biota nativa, devido ao acúmulo de biomassa vegetal seca.

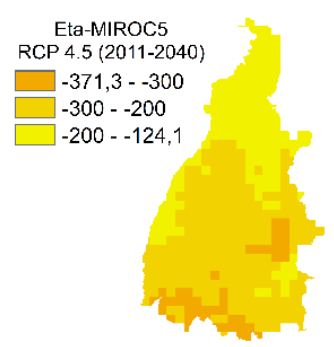

A

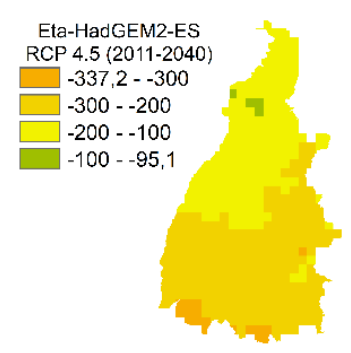

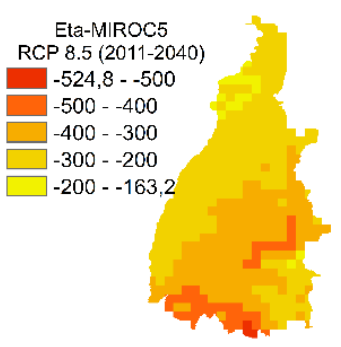

B

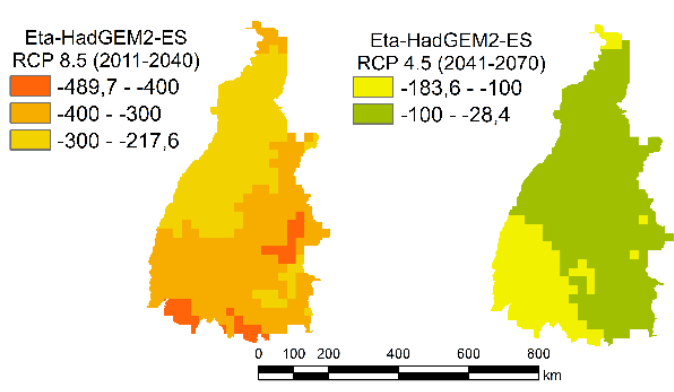

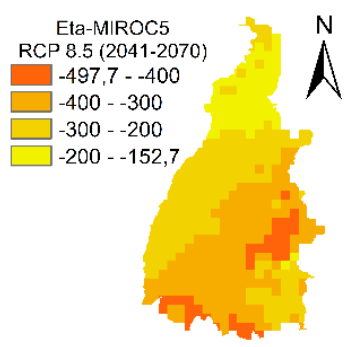

D

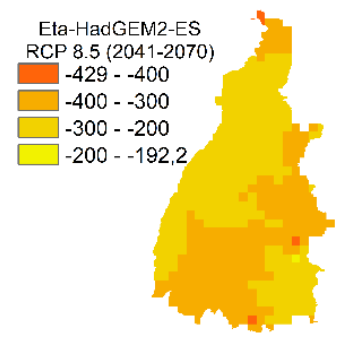

Figura 2 - Mudanças climáticas de precipitação, em mm. 6 meses $^{-1}$, projetadas para o período chuvoso (outubro a março) de 2011 a 2040 no RCP 4.5 (A) e RCP 8.5 (B), e de 2041 a 2070 no RCP 4.5 (C) e RCP 8.5 (D) pelo Eta-MIROC5 e Eta-HadGEM2-ES.

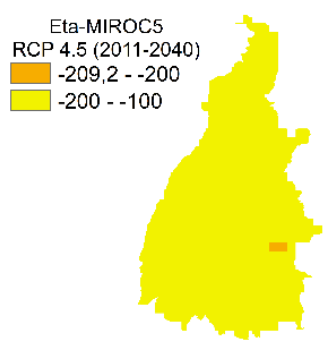

A

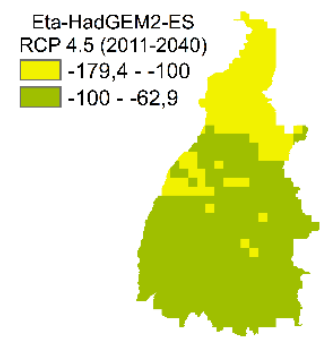

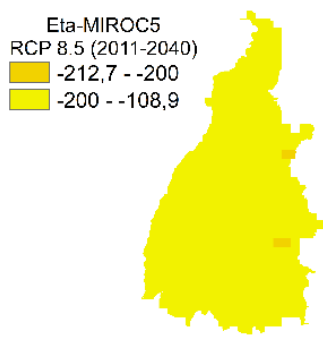

B

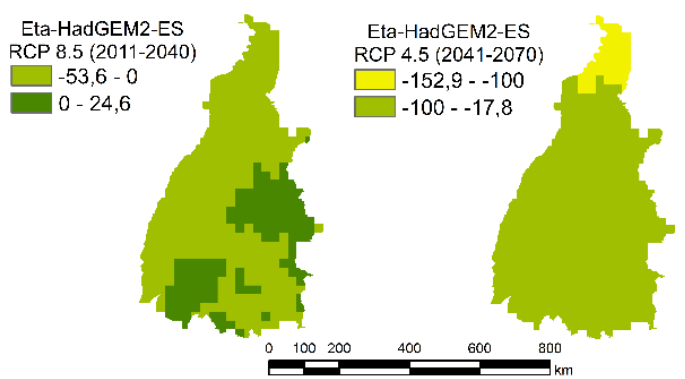

Eta-MIROC5 RCP $4.5(2041-2070)$
$-145,7--100$ $-100--73,3$

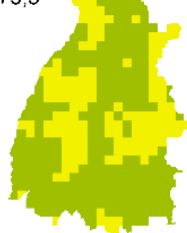

C

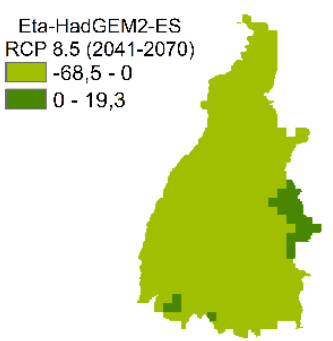

Figura 3 - Mudanças climáticas de precipitação, em mm. 6 meses $^{-1}$, projetadas para o período seco (abril a setembro) de 2011 a 2040 no RCP 4.5 (A) e 8.5 (B), e de 2041 a 2070 no RCP 4.5 (C) e 8.5 (D) pelo Eta-MIROC5 e Eta-HadGEM2-ES. 


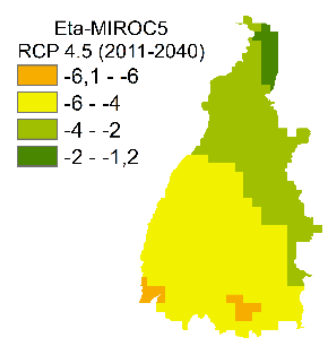

A

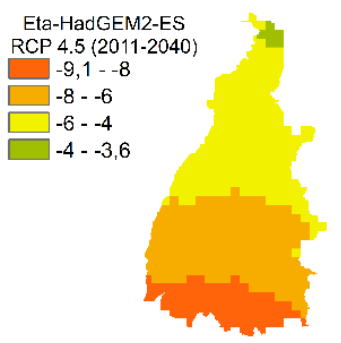

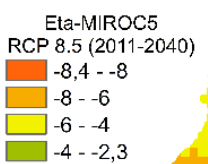

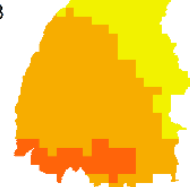

B

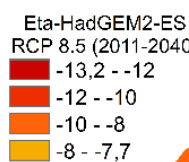

C

Eta-HadGEM2-ES RCP $4.5(2041-2070)$

$-6,1--6$
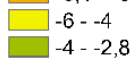
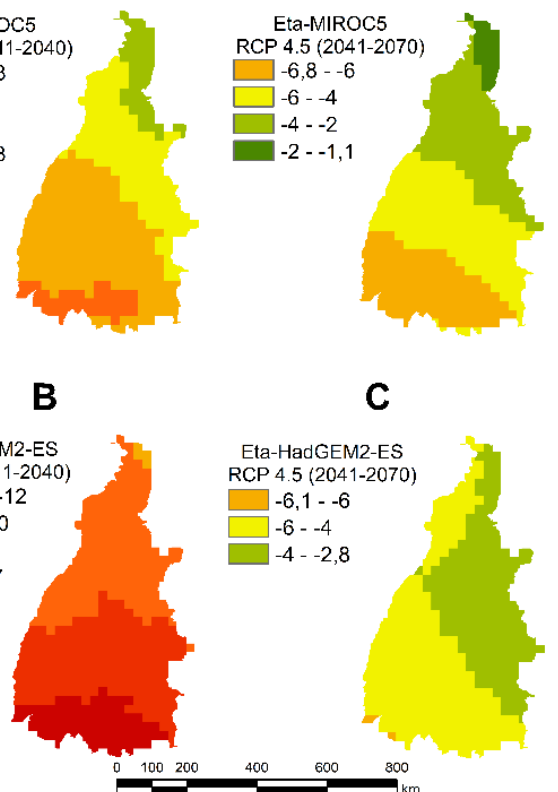

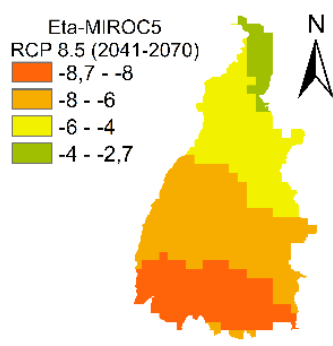

D

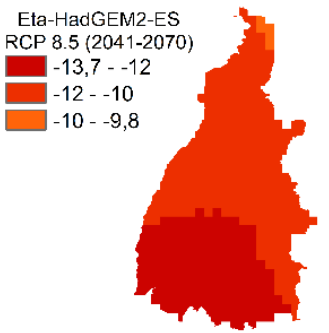

Figura 4 - Mudanças climáticas de umidade relativa, em \%, projetadas para o período chuvoso (outubro a março) de 2011 a 2040 no RCP 4.5 (A) e RCP 8.5 (B), e de 2041 a 2070 no RCP 4.5 (C) e RCP 8.5 (D) pelo Eta-MIROC5 e Eta-HadGEM2-ES.

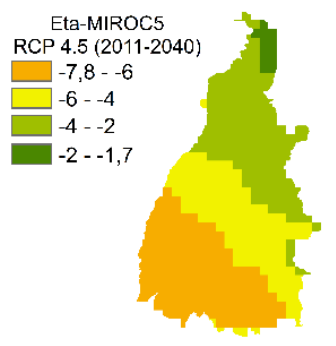

A

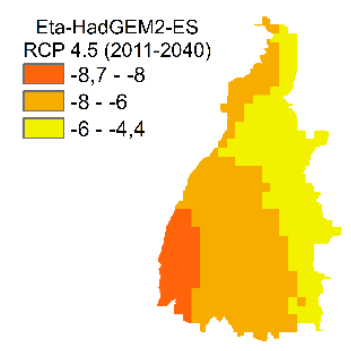

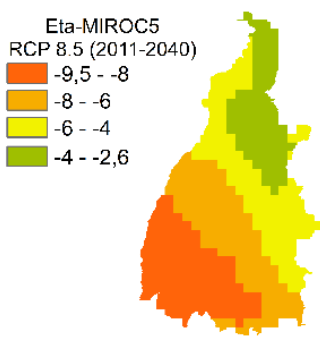

B

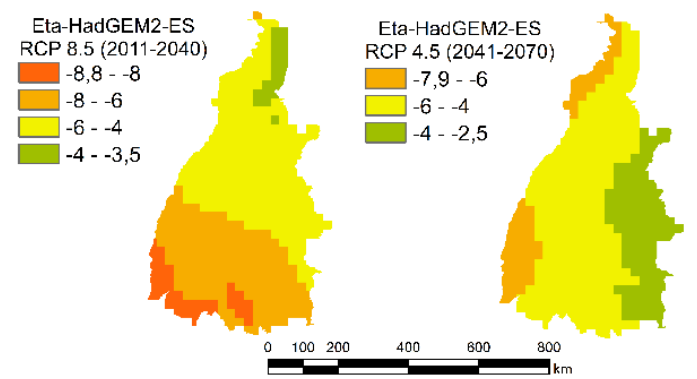

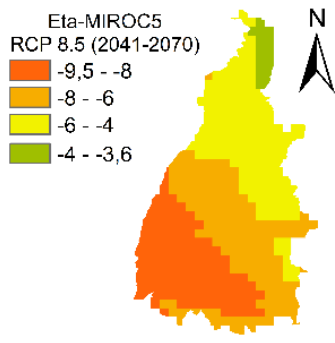

D

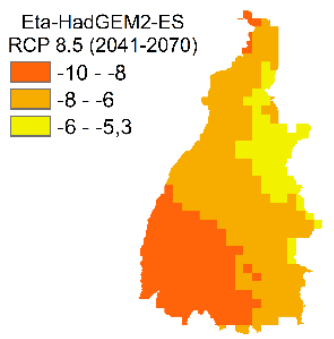

Figura 5 - Mudanças climáticas de umidade relativa, em \%, projetadas para o período seco (abril a setembro) de 2011 a 2040 no RCP 4.5 (A) e RCP 8.5 (B), e de 2041 a 2070 no RCP 4.5 (C) e RCP 8.5 (D) pelo Eta-MIROC5 e Eta-HadGEM2-ES.

As mudanças projetadas para as temperaturas média (Figuras 6 e 7), máxima (Figuras 8 e 9) e mínima (Figuras 10 e 11) apresentaram sinal positivo (aquecimento) em todas as análises, conforme também pode ser verificado na 
Tabela 1. As projeções mais severas foram obtidas pelo Eta-HadGEM2-ES no cenário RCP 8.5, para o segundo período futuro (2041-2070), sendo de até $5,1^{\circ} \mathrm{C}$ para a temperatura média, $5,9^{\circ} \mathrm{C}$ para temperatura máxima e $4,8^{\circ} \mathrm{C}$ para a temperatura mínima. De maneira geral, o aquecimento projetado foi persistente nos períodos chuvoso (out-mar) e estiagem (abr-set).

A distribuição espacial das mudanças projetadas para as temperaturas média, máxima e mínima, mostra um gradiente crescente na direção NordesteSudoeste, de maneira que as projeções mais severas se situam no Sudoeste. A projeção de aquecimento identificada corrobora para o aumento da evapotranspiração potencial. Associando a isto a projeção de redução da precipitação já abordada, têm-se um cenário pessimista do ponto de vista da gestão de recursos hídricos, de projeção de intensificação do déficit hídrico sobre o Cerrado tocantinense nos cenários RCP 4.5 e RCP 8.5. Rodrigues (2017) avaliou a evapotranspiração potencial nos cenários RCP 4.5 e RCP 8.5 em três bacias hidrográficas no sudeste do Tocantins. O autor verificou a possibilidade de aumento da evapotranspiração potencial em até 10,7\% e 19,1\% para o primeiro e segundo períodos futuros, respectivamente, o que está de acordo com as projeções avaliadas no presente estudo.

Cabe mencionar que as projeções de mudanças na temperatura pelo EtaMIROC5 foram menores que as do Eta-HadGEM2-ES, não ultrapassando $3,4^{\circ} \mathrm{C}$ (temp. média), $3,9^{\circ} \mathrm{C}$ (temp. máxima) e $2,8^{\circ} \mathrm{C}$ (temp. mínima).

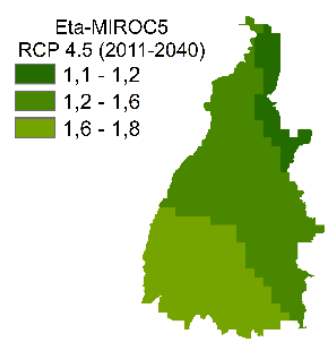

A

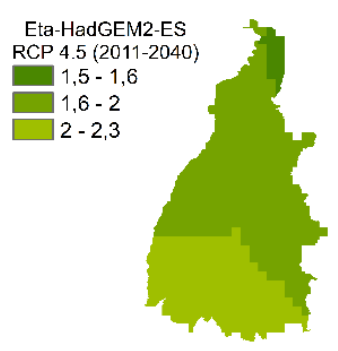

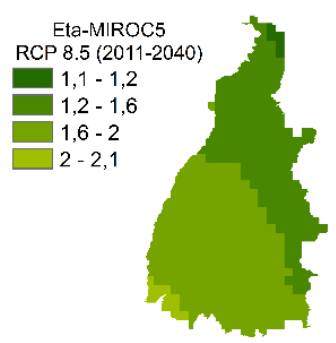

B
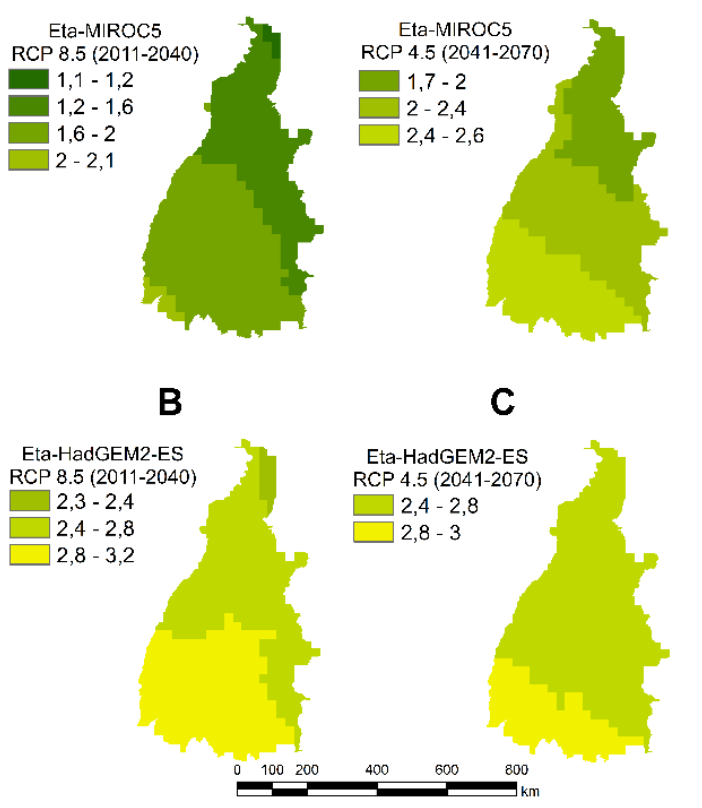

C

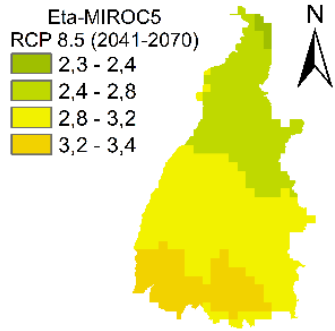

D

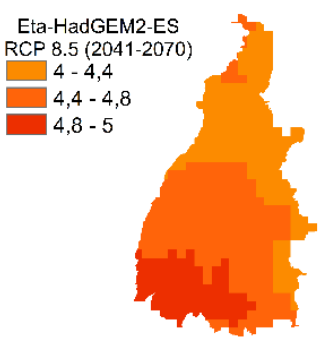

Figura 6 - Mudanças climáticas de temperatura média, em ${ }^{\circ} \mathrm{C}$, projetadas para $\mathrm{O}$ período chuvoso (outubro a março) de 2011 a 2040 no RCP 4.5 (A) e 8.5 (B), e de 2041 a 2070 no RCP 4.5 (C) e 8.5 (D) pelo Eta-MIROC5 e Eta-HadGEM2-ES. 


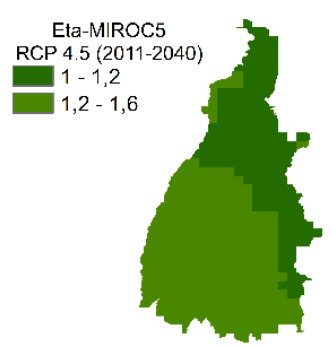

A

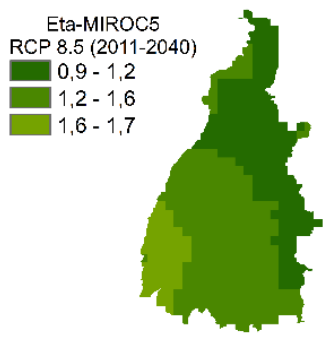

B

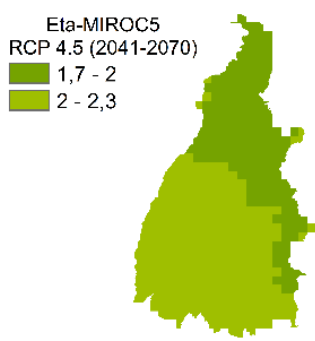

C

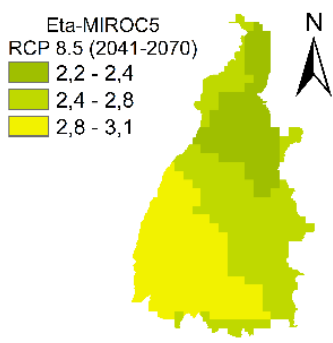

D
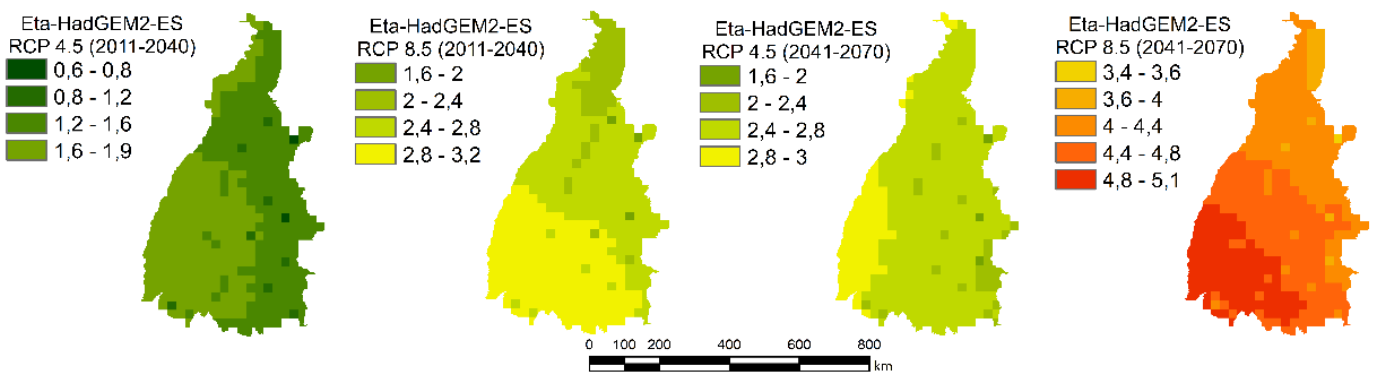

Figura 7 - Mudanças climáticas de temperatura média, em ${ }^{\circ} \mathrm{C}$, projetadas para o período seco (abril a setembro) de 2011 a 2040 no RCP 4.5 (A) e 8.5 (B), e de 2041 a 2070 no RCP 4.5 (C) e 8.5 (D) pelo Eta-MIROC5 e Eta-HadGEM2-ES.

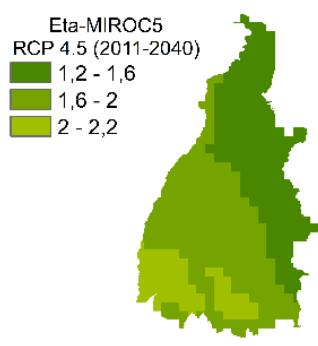

A

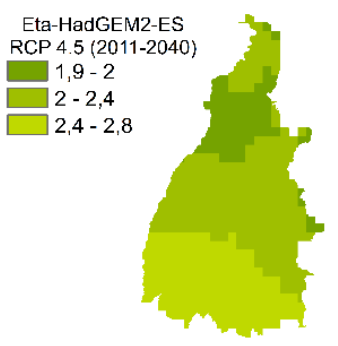

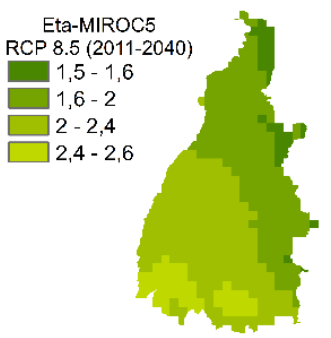

B

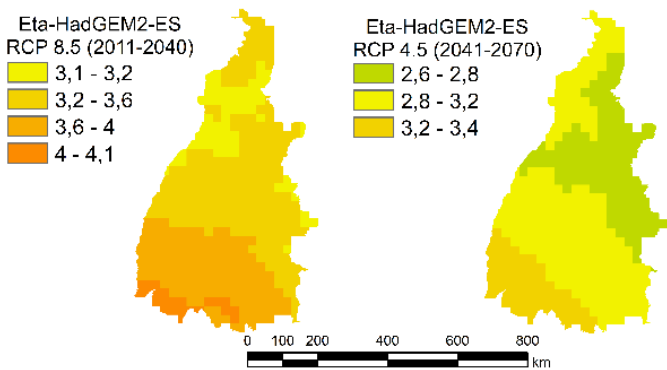

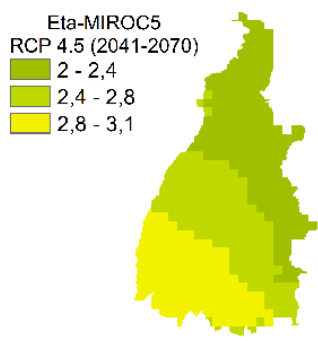

C

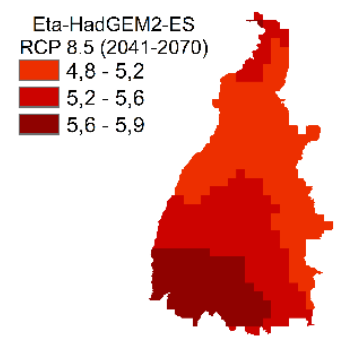

Figura 8 - Mudanças climáticas de temperatura máxima, em ${ }^{\circ} \mathrm{C}$, projetadas para $\mathrm{O}$ período chuvoso (outubro a março) de 2011 a 2040 no RCP 4.5 (A) e 8.5 (B), e de 2041 a 2070 no RCP 4.5 (C) e 8.5 (D) pelo Eta-MIROC5 e Eta-HadGEM2-ES. 


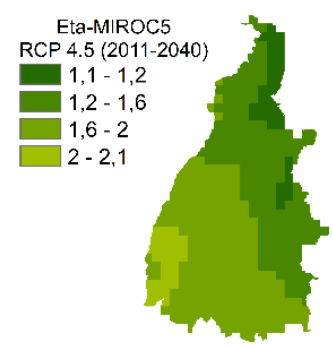

A

Eta-HadGEM2-ES RCP $4.5(2011-2040)$

$1,7-2$

$2-2,4$

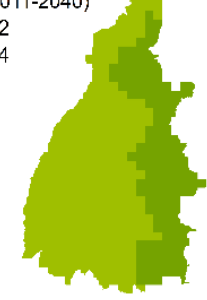

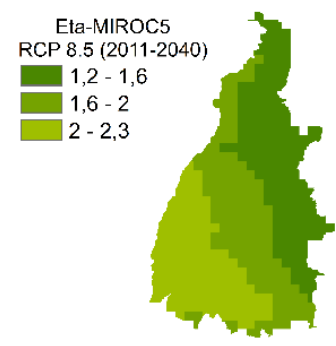

B

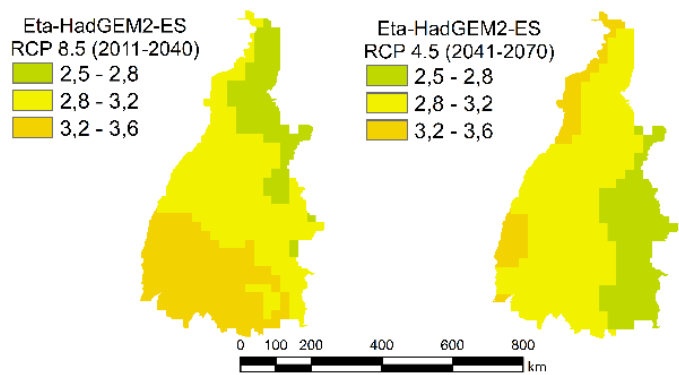

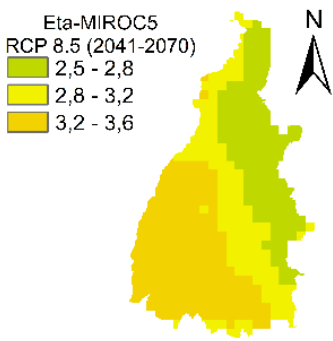

D

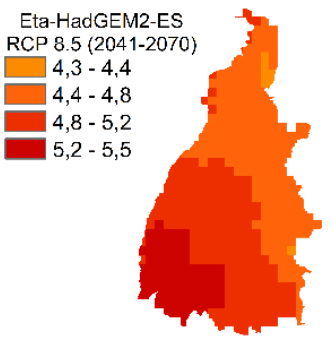

Figura 9 - Mudanças climáticas de temperatura máxima, em ${ }^{\circ} \mathrm{C}$, projetadas para $\mathrm{O}$ período seco (abril a setembro) de 2011 a 2040 no RCP 4.5 (A) e 8.5 (B), e de 2041 a 2070 no RCP 4.5 (C) e 8.5 (D) pelo Eta-MIROC5 e Eta-HadGEM2-ES.

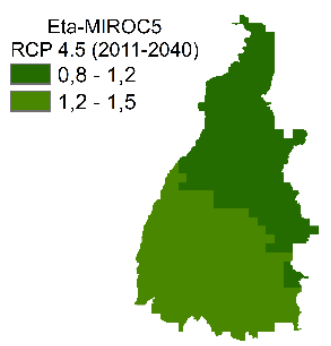

A

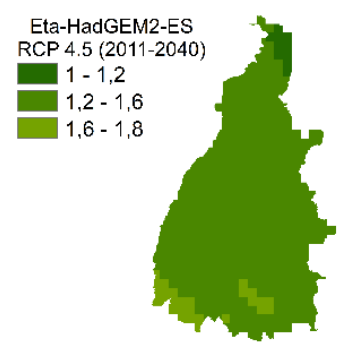

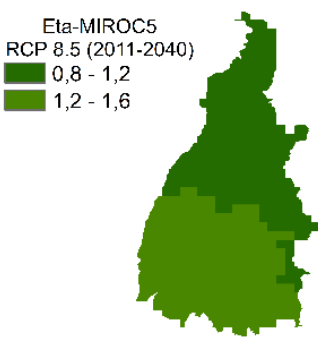

B

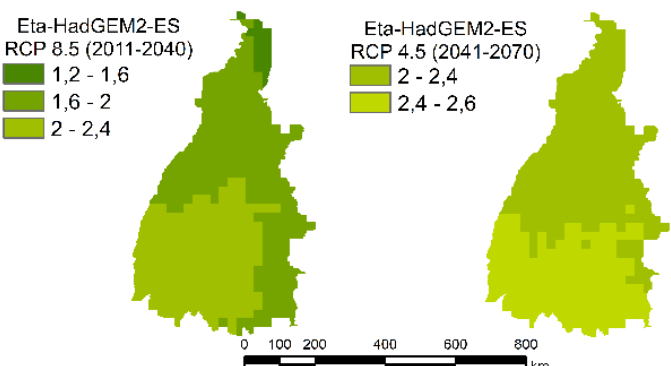

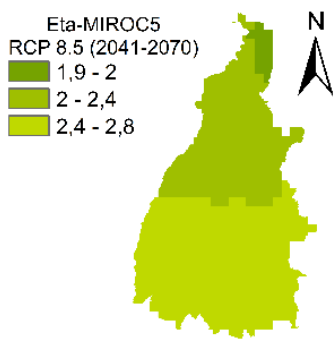

D

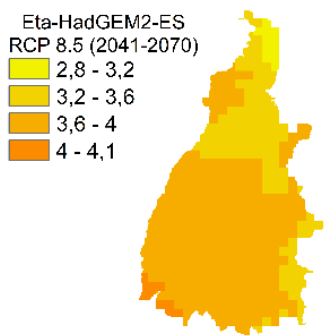

Figura 10 - Mudanças climáticas de temperatura mínima, em ${ }^{\circ} \mathrm{C}$, projetadas para 0 período chuvoso (outubro a março) de 2011 a 2040 no RCP 4.5 (A) e 8.5 (B), e de 2041 a 2070 no RCP 4.5 (C) e 8.5 (D) pelo Eta-MIROC5 e Eta-HadGEM2-ES. 


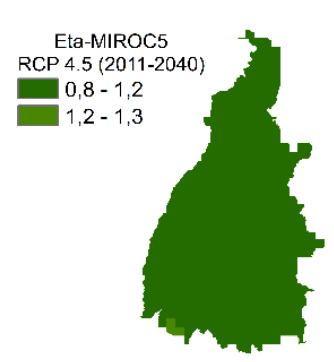

A

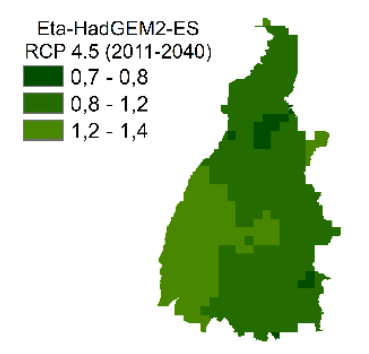

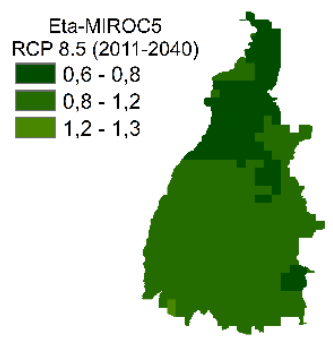

B

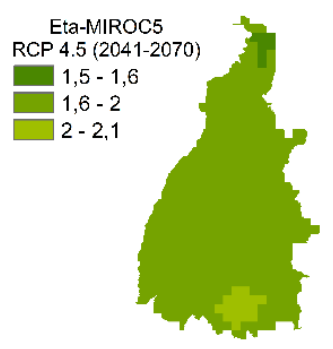

C

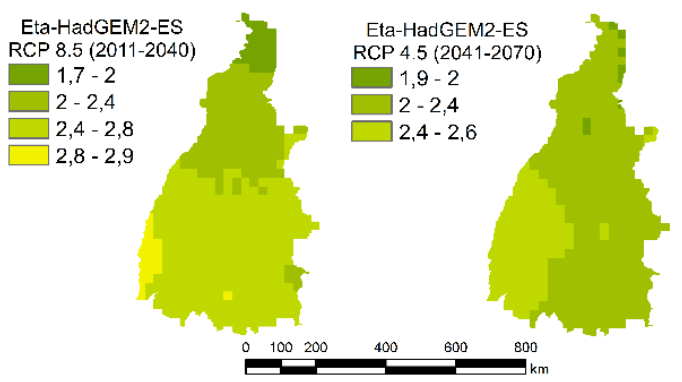

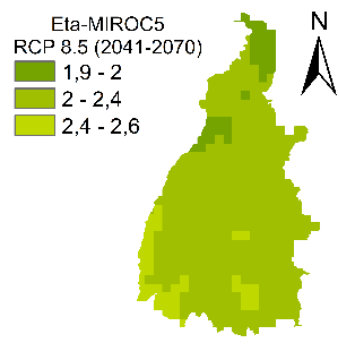

D

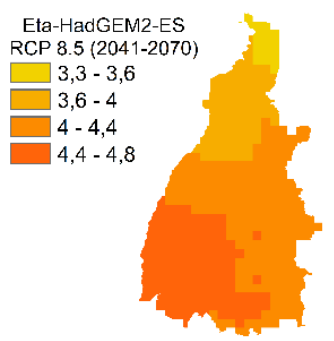

Figura 11 - Mudanças climáticas de temperatura mínima, em ${ }^{\circ} \mathrm{C}$, projetadas para 0 período seco (abril a setembro) de 2011 a 2040 no RCP 4.5 (A) e 8.5 (B), e de 2041 a 2070 no RCP 4.5 (C) e 8.5 (D) pelo Eta-MIROC5 e Eta-HadGEM2-ES.

As mudanças climáticas futuras projetadas para a evapotranspiração real (Figuras 12 e 13), apresentaram amplitude de variação nos intervalos de [$\left.206,7 \mathrm{~mm} .6 \mathrm{meses}^{-1} ;-28,1 \mathrm{~mm} \cdot 6 \mathrm{meses}^{-1}\right]$ e de $\left[-189,7 \mathrm{~mm} \cdot 6 \mathrm{meses}^{-1} ;-4,4\right.$ $\mathrm{mm} .6 \mathrm{meses}^{-1}$ ] para o primeiro e segundo período futuro, respectivamente (Tabela 1). Para o período chuvoso as projeções mais severas se localizaram no Sudeste, enquanto que, para o período de estiagem estas se localizaram no Sudoeste. Tais resultados projetados pelos modelos climáticos encontram subsídio nas projeções de aumento de temperatura e de redução dos totais precipitados, anteriormente abordadas. O Eta-HadGEM2-ES no RCP 8.5 resultou nas mudanças mais severas projetadas para esta análise. Rodrigues (2017) aplicou o modelo hidrológico Soil and Water Assessment Tool (SWAT) para avaliar os possíveis impactos hidrológicos futuros em três bacias hidrográficas no sudeste do Tocantins frente aos cenários RCP 4.5 e RCP 8.5 projetados pelo Eta-MIROC5 e Eta-HadGEM2-ES. O autor verificou possibilidade de redução da disponibilidade hídrica para ambos RCPs nas três bacias, sendo a redução mais severa obtida pelo Eta-HadGEM2-ES, RCP8.5.

Observa-se também, pela análise dos resultados das projeções climáticas para o Estado do Tocantins, que o RCP pessimista (8.5) foi mais impactante do que o RCP intermediário (4.5). Isto evidencia que o desenvolvimento de políticas públicas visando a redução de emissão de GEEs de origem antrópica pode corroborar para mitigação de possíveis impactos sobre a disponibilidade hídrica.

Em síntese, a análise de possíveis mudanças climáticas para o século XXI no estado do Tocantins mostrou concordância entre as projeções dos MCGs (HadGEM2-ES e MIROC5) regionalizadas pelo MCR Eta. As projeções de aumento da temperatura e de redução da precipitação para o século XXI tem 
como um dos possíveis impactos a redução da disponibilidade hídrica, visto que o saldo do balanço hídrico vertical tende a ser reduzido nesta situação.

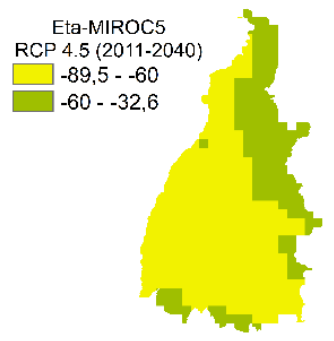

A

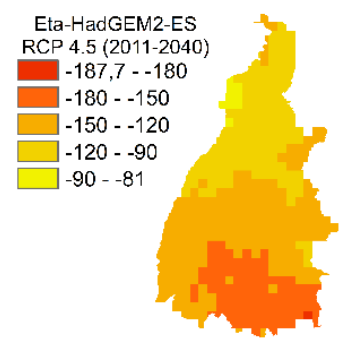

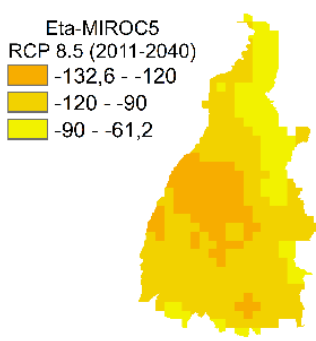

B

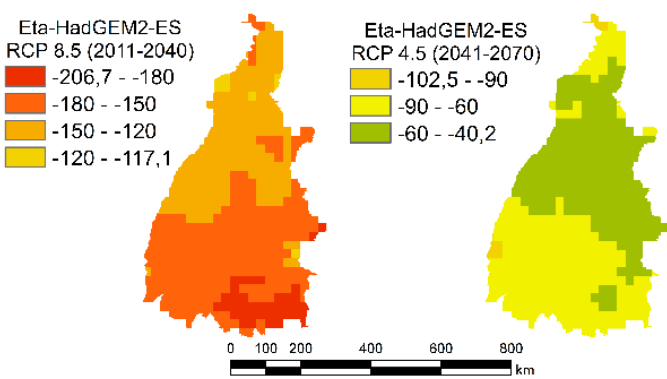

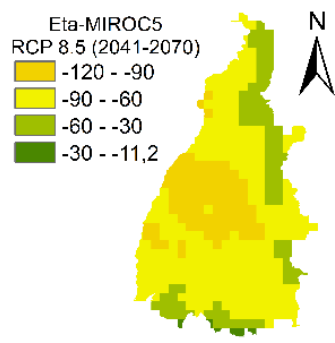

D

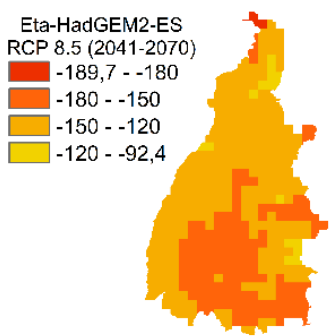

Figura 12 - Mudanças climáticas de evapotranspiração, em mm.6meses ${ }^{-1}$, projetadas para o período chuvoso (outubro a março) de 2011 a 2040 no RCP 4.5 (A) e 8.5 (B), e de 2041 a 2070 no RCP 4.5 (C) e 8.5 (D) pelo Eta-MIROC5 e Eta-HadGEM2-ES.

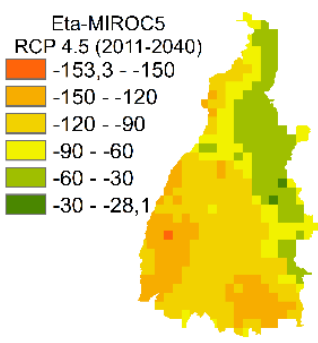

A

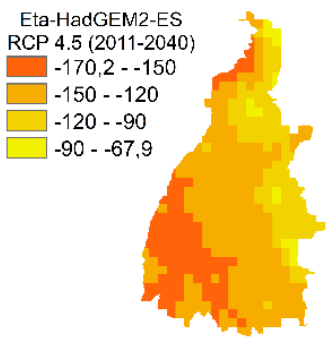

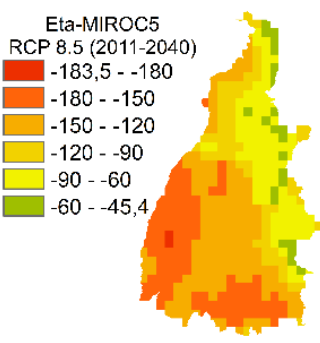

B

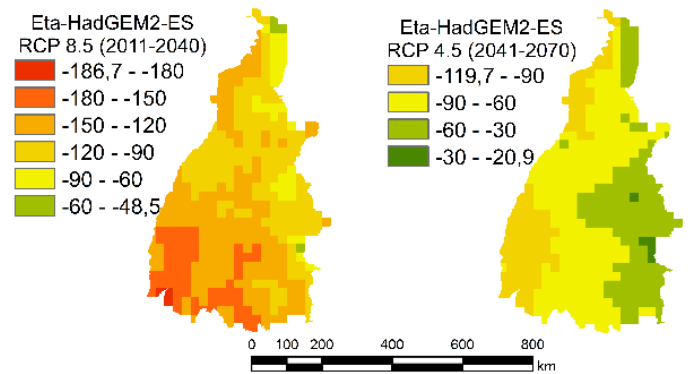

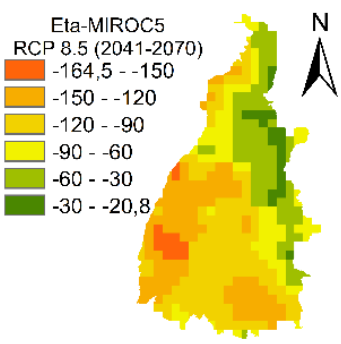

D

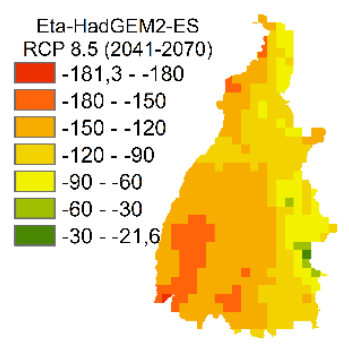

Figura 13 - Mudanças climáticas de evapotranspiração, em mm.6meses ${ }^{-1}$, projetadas para o período seco (abril a setembro) de 2011 a 2040 no RCP 4.5 (A) e 8.5 (B), e de 2041 a 2070 no RCP 4.5 (C) e 8.5 (D) pelo Eta-MIROC5 e Eta-HadGEM2-ES. 


\section{CONCLUSÕES}

As projeções de mudanças climáticas futuras de precipitação pelo EtaHadGEM2-ES e Eta-MIROC5 mostraram possibilidade de redução da precipitação nos cenários RCP 4.5 e RCP 8.5 para o estado do Tocantins, sobretudo para a região sul. Destaca-se a mudança mais severa para a precipitação projetada pelo Eta-MIROC5 no RCP 8.5, de até $-524,8$ mm.6meses ${ }^{-1}$ (out-mar) para o início do século XXI (2011-2040).

As projeções climáticas futuras mostraram possibilidade de aquecimento no estado do Tocantins nos cenários RCP 4.5 e RCP 8.5. A simulação mais severa foi obtida pelo modelo regional Eta dirigido pelo MCG HadGEM2-ES no RCP 8.5 para a metade do século XXI (2041-2070). As localidades com projeções de maior aquecimento se encontram no sudoeste do Estado, na região da Ilha do Bananal e APA dos Meandros do rio Araguaia. Para as temperaturas média, máxima e mínima, as projeções foram de aumento de até $5,1^{\circ} \mathrm{C}, 5,9^{\circ} \mathrm{C}$ e $4,8^{\circ} \mathrm{C}$, respectivamente.

Os possíveis impactos sobre a disponibilidade hídrica decorrentes das projeções de aumento de temperatura e de redução da precipitação nos cenários RCP 4.5 e RCP 8.5, permitem concluir sobre a necessidade de planejamento de medidas mitigadoras pela gestão de recursos hídricos.

\section{AGRADECIMENTOS}

Os autores agradecem ao Conselho Nacional de Desenvolvimento Científico e Tecnológico (CNPq) pelo apoio aos projetos 482075/2013-9 e 308947/2018-5.

\section{REFERÊNCIAS BIBLIOGRÁFICAS}

ADAM, K. N.; COLLISCHONN, W. Análise dos impactos de mudanças climáticas nos regimes de precipitação e vazão na bacia hidrográfica do rio Ibicuí. Revista Brasileira de Recursos Hídricos, v. 18, n. 3, p. 69-79, jul/set. 2013.

BLACK, T. L. The new NMC mesoescale Eta model. Description and forecast examples. Weather and Forecasting, v. 9, n. 2, p. 265-278, jun. 1994.

CHOU, S, C.; MARENGO, J. A.; LYRA, A. A.; SUEIRO, G.; PESQUERO, J. ; ALVES, L. M.; KAY, G.; BETTS, R.; CHAGAS, D.; GOMES, J. L.; BUSTAMANTE, J.; TAVARES, P. Downscaling of South America present climate driven by 4-member HadCM3 runs. Climate Dynamics, v.38, n. 3, p. 635-653, fev. 2012

CHOU, S. C.; LYRA, A.; MOURÃO, C.; DERECZYNSKI, C.; PILOTTO, I.; GOMES, J.; BUSTAMANTE, J.; TAVARES, P.; SILVA, A.; RODRIGUES, D.; CAMPOS, D.; SUEIRO, G.; SIQUEIRA, G.; NOBRE, P.; MARENGO, J. Evaluation of the Eta simulations nested in three global climate models. American Journal of Climate Change, v. 3, n. 5, p. 438-454, dez. 2014a.

CHOU, S. C.; LYRA, A.; MOURÃO, C.; DERECZYNSKI, C.; PILOTTO, I. ; GOMES, J.; BUSTAMANTE, J.; TAVARES, P.; SILVA, A.; RODRIGUES, D.; CAMPOS, D.; CHAGAS, D.; SUEIRO, G.; SIQUEIRA, G. MARENGO, J. Assessment of climate change over South America under RCP 4.5 and 8.5 downscaling scenarios. American Journal of Climate Change, v. 3, p. 512-525, dez. 2014b. 
COLLINS, W. J.; BELLOUIN, N.; DOUTRIAUX-BOUCHER, M.; GEDNEY, N.; HALLORAN, P.; HINTON, T.; HUGHES, J.; JONES, C. D.; JOSHI, M.; LIDDICOAT, S.; MARTIN, G.; O'CONNOR, F.; RAE, J.; SENIOR, C.; SITCH, S.; TOTTERDELL, I.; WILTSHIRE, A.; WOODWARD, S. Development and Evaluation of an EarthSystem Model-HadGEM2. Geoscientific Model Development, v. 4, n. 4, p. 10511075, nov. 2011.

COX P. M.; BETTS R. A.; COLLINS, M.; HARRIS, P. P.; HUNTINGFORD, C.; JONES C. D. Amazonian forest dieback under climate-carbon cycle projections for the 21st century. Theoretical and Applied Climatology, v. 78, n. 1-3, p. 137156, jun. 2004.

CUBASCH, U.; WUEBBLES, D.; CHEN, D.; FACCHINI, M. C.; FRAME, D.; MAHOWALD, N.; WINTHER, J-G. Introduction. In: Climate Change 2013: Climate Change 2013: The Physical Science Basis. Contribution of Working Group I to the Fifth Assessment Report of the Intergovernmental Panel on Climate Change. Cambridge University Press, Cambridge. p. 119-158, 2013.

DELAZERI, L. M. M. Mudanças climáticas e migração rural-urbana no Semiárido brasileiro. 2015. 73 f. Dissertação (Mestrado em Economia Aplicada) Universidade Federal de Viçosa, Viçosa, 2015.

EVANGELISTA, L. B. Relações socioeconômicas e ambientais no Cerrado: o cenário goiano. 2016. 50 f. Dissertação (Mestrado em Ciências Ambientais e Saúde) - Universidade Católica de Goiás, Goiânia, 2016.

FELFILI, J. M.; SILVA-JUNIOR, M. C.; SEVILHA, A. C.; FAGG, C. W.; WALTER, B. M. T.; NOGUEIRA, P. E.; REZENDE, A. V. Diversity, floristic and structural patterns of cerrado vegetation in Central Brazil. Plant Ecology, v. 175, n. 1, p. 37-46, jul. 2004.

FESER, F.; ROCKEL, B.; STORCH, H. S.; WINTERFELDT, J.; ZAHN, M. Regional climate models add value to global model data: A review and selected examples. American Meteorological Society, v. 92, p. 1181-1192, set. 2011.

FRANÇOSO, R. D; HAIDAR, R. F; MACHADO, R. B. Tree species of South America central savanna: endemism, marginal areas, the relationship with other biomes. Acta Botanica Brasilica, v. 30, n. 1, p. 78-86, jan-mar.2016.

GOLDING, N.; BETTS R. Fire risk in Amazonia due to climate change in the HadCM3 climate model: Potential interactions with deforestation. Global Biogeochemical Cycles, v. 22, n. 4, GB4007, 2008.

HASUMI, H. CCSR Ocean Component Model (COCO), version 4.0. Center for Climate System Research Rep. 25, 2006, 103 p. Disponível em: < http://ccsr.aori.u-tokyo.ac.jp/ hasumi/COCO/coco4.pdf> Acesso em: 12 nov. 2016.

IBGE. Instituto Brasileiro de Geografia e Estatística. Mapa dos biomas do Brasil. Escala 1: 5.000.000. 2004. Disponível em: <http://mapas.ibge.gov.br/biomas2/viewer.htm>. Acesso em: 23 nov. 2016.

IBGE. Instituto Brasileiro de Geografia e Estatística. Estados@ Tocantins. 2016. Disponível em: < http://www.ibge.gov.br/estadosat/perfil.php?sigla=to>. Acesso em: 15 jul. 2017.

IPCC. Intergovernmental Panel on Climate Change. Climate Change 2013: The Physical Science Basis. Contribution of Working Group I to the Fifth Assessment 
Report of the Intergovernmental Panel on Climate Change. Cambridge University Press, Cambridge, United Kingdom and New York, NY, USA, 2013, $1535 \mathrm{p}$.

IPCC. Intergovernmental Panel on Climate Change. Climate Change 2013: The Physical Science Basis. Contribution of Working Group I to the Fourth Assessment Report of the Intergovernmental Panel on Climate Change. Cambridge University Press, Cambridge, United Kingdom and New York, NY, USA, 2007, 996 p.

KLINK, C. A.; MOREIRA, A. G. Past and current human occupation, and land use. In: Oliveira, p.S.; Marquis, R.J. (eds.) The Cerrados of Brazil: ecology and natural history of Neotropical savanna. New York: Columbia University Press, cap. 5, p. 69-90, 2002.

LIMA, J. E. F. W.; SILVA, E. M. Estimativa da contribuição hídrica superficial do Cerrado para as grandes regiões hidrográficas brasileiras. In: Anais do XVII Simpósio Brasileiro de Recursos Hídricos, 2007, São Paulo: ABRH, 2007.

LIMA, J. E. F. W. Situação e Perspectivas Sobre as Águas do Cerrado. Ciência e Cultura, v. 63, n. 3, p. 27-29, jul. 2011.

MALHI, Y., ARAGAO, L. E. O. C., GALBRAITH, D., HUNTINGFORD, C., FISHER, R., ZELAZOWSKI, P., ... MEIR, P. Exploring the likelihood and mechanism of a climate-change-induced dieback of the Amazon rainforest. Proceedings of the National Academy of Sciences of the United States of America - PNAS, v. 106, n. 49, p. 20610-20615, 2009.

MARENGO, J. A.; CHOU, S. C.; KAY, G.; ALVES, L. M.; PESQUERO, J. F.; SOARES, W. R.; SANTOS, D. C.; LYRA, A. A.; SUEIRO, G.; BETTS, R.; CHAGAS, D. J.; GOMES, J. L.; BUSTAMANTE, J. F.; TAVARES P. Development of regional future climate change scenarios in South America using the Eta CPTEC/HadCM3 climate change projections: Climatology and regional analyses for the Amazon, São Francisco and the Paraná River Basins. Climate Dynamics, v. 38, n. 9-10, p. 1829-1848, 2012.

MARENGO, J. A.; NUNES, L. H.; SOUZA, C. R. G.; HARARI, J.; MULLER-KARGER, F.; GRECO, R.; HOSOKAWA, E. K.;TABUCHI, E. K.; MERRILL, S. B.; REYNOLDS, C. J.; PELLING, M.; ALVES, L. M.; ARAGÃO, L. E.; CHOU, S. C.; MOREIRA, F.; PATERSON, S.; LOCKMAN, J. T.; GRAY, A. G. A globally deployable strategy for co-development of adaptation preferences to sea-level rise: the public participation case of Santos, Brazil. Natural Hazards, v. 88, n. 1, p.39-53, abr. 2017.

MARTIN, G. M.; BELLOUIN, N.; COLLINS, W. J.; CULVERWELL, I. D.; HALLORAN, P. R.; HARDIMAN, S. C.; HINTON, T. J.; JONES, C. D.; MCDONALD, R. E.; MCLAREN, A. J.; O'CONNOR, F. M.; ROBERTS, M. J.; RODRIGUEZ, J. M.; WOODWARD, S.; BEST, M. J.; BROOKS, M. E.; BROWN, A. R.; BUTCHART, N.; DEARDEN, C.; DERBYSHIRE, S. H.; DHARSSI, I.; DOUTRIAUX-BOUCHER, M.; EDWARDS, J. M.; FALLOON, P.D.; GEDNEY, N.; GRAY, L. J.; HEWITT, H. T.; HOBSON, M.; HUDDLESTON, M. R.; HUGHES, J.; INESON, S.; INGRAM, W. J.; JAMES, P. M.; JOHNS, T. C.; JOHNSON, C. E.; JONES, A.; JONES, C. P.; JOSHI, M. M.; KEEN, A. B.; LIDDICOAT, S.; LOCK, A. P.; MAIDENS, A. V.; MANNERS, J. C.; MILTON, S. F.; RAE, J. G. L.; RIDLEY, J. K.; SELLAR, A.; SENIOR, C. A.; TOTTERDELL, I. J.; VERHOEF, A.; VIDALE, P. L.; WILTSHIRE A. The HadGEM2 
family of Met Office Unified Model climate configurations. Geoscientific Model Development, v. 4, n. 3, p. 723-757, set. 2011.

MELO, A. S.; JUSTINO, F. B.; MELO, E. C. S.; SILVA, T. L. V. Índices de risco de fogo de haines e setzer em diferentes condições climáticas. Mercator. v. 11, $\mathrm{n}$. 24, p. 187-207, jan./abr. 2012.

MENDONÇA, R. C.; FELFILI, J. M; WALTER B. M. T.; SILVA JÚNIOR, M. C.; REZENDE, A. V.; FILGUEIRAS, T. S.; NOGUEIRA, P. E.; FAGG, C. W. Flora Vascular do bioma Cerrado: checklist com 12.356 espécies. In Cerrado: ecologia e flora (SANO, S. M.; ALMEIDA, S. P; RIBEIRO, J. F. EDS.). Embrapa Cerrados, Planaltina, p. 421-1279, 2008.

MESINGER, F.; CHOU, S. C.; GOMES, J. L.; JOVIC, D.; BASTOS, P. R.; BUSTAMANTE, J. F. ; LAZIC, L.; LYRA, A. A.; MORELLI, S.; RISTIC, I. ; VELJOVIC, K. An upgraded version of Eta Model. Meteorology and Atmospheric Physics, $v$. 116, n. 3, p. 63-79, mai. 2012.

MOSS, R. H.; EDMONDS, J. A.; HIBBARD, K. A.; MANNING, M. R.; ROSE, S.K.; VAN VUUREN, D. P.; CARTER, T. R.; EMORI, S.; KAINUMA, M.; KRAM, T.; MEEHL, G. A.; MITCHELL, J. F.; NAKICENOVIC, N.; RIAHI, K.; SMITH, S. J.; STOUFFER, R. J.; THOMSON, A. M.; WEYANT, J. P.; WILBANKS, T. J. The next generation of scenarios for climate change research and assessment. Nature, $v$. 463, n. 7282, p. 747-756, fev. 2010.

PESQUERO, J. F.; CHOU, S. C.; NOBRE, C. A.; MARENGO, J. A. Climate downscaling over South America for 1961-1970 using the Eta Model. Theoretical and Applied Climatology, v. 99, n. 1-2, p. 75-93, jan. 2010.

RIAHI, K.; RAO, S.; KREY, V.; CHO, C.; CHIRKOV, V.; FISCHER, G.; RAFAJ, P. RCP 8.5 - A scenario of comparatively high greenhouse gas emissions. Climatic Change, v. 109, n. 1, p. 33-57, nov. 2011.

RODRIGUES, J. A. M. Impactos antrópicos no regime hidrológico de tributários do Rio Tocantins. 2017. 128 f. Dissertação (Mestrado em Recursos Hídricos em Sistemas Agrícolas) - Universidade Federal de Lavras, Lavras, MG, 2017.

SILVEIRA, C. S.; SOUZA FILHO, F. A.; MARTINS, E. S. P. R., OLIVEIRA, J. L.; COSTA, A. C.; NOBREGA, M. T.; SOUZA, S. A.; SILVA, R. F. V. Mudanças climáticas na bacia do rio São Francisco: Uma análise para precipitação e temperatura. Revista Brasileira de Recursos Hídricos, v. 21, n. 2, p. 416 - 428, 2016.

SOUZA, F. H. M.; VIOLA, M. R.; AVANZI, J. C.; GIONGO, M.; VIEIRA FILHO, M. Regionalização climática de Thornthwaite para o Estado de Tocantins. Rev Floresta. v. 49, n.4, set. 2019.

TAN, M. L.; IBRAHIM, A. L.; YUSOP, Z.; CHUA, V. P.; CHAN, N. W. Climate change impacts under CMIP5 RCP scenarios on water resources of the Kelantan River Basin, Malaysia. Atmospheric Research. V. 189, p. 1-10, jan. 2017,

THOMSON, A. M; CALVIN, K. V; SMITH, S. J; KYLE, G. P; VOLKE, A; PATEL, P.; DELGADO-ARIAS, S.; BOND-LAMBERTY, B.; WISE, M. A.; CLARKE, L. E.; EDMONDS, J. A. RCP 4.5: a pathway for stabilization of radiative forcing by 2100. Climatic Change, v. 109, n.1, p. 77-94, nov. 2011. 
WANG, T. L.; CAMPBELL, E. M.; O'NEILL, G. A.; AITKEN, S. N. Projecting future distributions of ecosystem climate niches: Uncertainties and management applications. Forest Ecology and Management, v. 279, p. 128-140, jan. 2012.

WATANABE, M.; SUZUKI, T.; O'ISHI, R.; KOMURO, Y.; WATANABE, S.; EMORI, S.; TAKEMURA, T.; CHIKIRA, M.; OGURA, T.; SEKIGUCHI, M.; TAKATA, K.; YAMAZAKI, D.; YOKOHATA, T.; NOZAWA, T.; HASUMI, H.; TETEBE, H.; KIMOTO, M. Improved climate simulation by MIROC5: mean states, variability, and climate sensitivity. Journal of Climate, v. 23, n. 23. p. 6312-6335, dez. 2010.

VAN VUUREN, D. P.; KAINUMA, M.; RIAHI, K.; THOMSON, A.; HIBBARD, K.; HURTT, G. C.; RAM, T.; KREY, V.; LAMARQUE, J.; MASUI, T.; MEINSHAUSEN, M.; NAKICENOVIC, N.; SMITH, S. J.; ROSE, S. K. The representative concentration pathways: An overview. Climate Change, v. 109, n. 1-2, p. 5-31, nov. 2011 